\title{
Multiscale modelling couples patches of wave-like simulations
}

\author{
Meng $\mathrm{Cao}^{1} \quad$ A. J. Roberts ${ }^{2}$
}

(Received 3 October 2012; revised 21 March 2013)

\begin{abstract}
A multiscale model is proposed to significantly reduce the expensive numerical simulations of complicated waves over large spatial domains. The multiscale model is built from given microscale simulations of complicated physical processes such as sea ice or turbulent shallow water. Our long term aim is to enable macroscale simulations obtained by coupling small patches of simulations together over large physical distances. This initial work explores the coupling of patch simulations of wave-like PDEs. With the line of development being to water waves we discuss the dynamics of two complementary fields called the 'depth' and 'velocity'. A staggered grid is used for the microscale simulation of the depth and velocity. We introduce a macroscale staggered grid to couple the microscale patches. Linear or quadratic interpolation
\end{abstract}

http://journal . austms.org.au/ojs/index.php/ANZIAMJ/article/view/6137 gives this article, (C) Austral. Mathematical Soc. 2013. Published May 14, 2013, as part of the Proceedings of the 16th Biennial Computational Techniques and Applications Conference. ISSN 1446-8735. (Print two pages per sheet of paper.) Copies of this article must not be made otherwise available on the internet; instead link directly to this URL for this article. 
provides boundary conditions on the field in each patch. Linear analysis of the whole coupled multiscale system establishes that the resultant macroscale dynamics is appropriate. Numerical simulations support the linear analysis. This multiscale method should empower the feasible computation of large scale simulations of wave-like dynamics with complicated underlying physics.

\section{Contents}

1 Introduction

2 The microscale simulator

C157

3 Couple microscale patches across gaps

C159

4 Linear analysis of the coupled dynamics

C163

5 Conclusion

C167

References

C168

\section{Introduction}

Numerical simulations of complicated waves over large spatial domains including tsunamis, floods and rivers are expensive and complex. Many researchers are exploring multiscale models to improve the accuracy and reduce the expense of such large scale simulations (Hou et al., 2008; Hu, 2012; Kevrekidis et al., 2003; Roberts and Kevrekidis, 2005, e.g.). The gap-tooth scheme was introduced and reviewed by Kevrekidis et al. (2003) and Samaey et al. (2005, 2009). The scheme uses microscale simulations on small patches of space, coupling the simulations over the intervening space, to simulate the system 
over a macroscale. The gap-tooth method adapts to whatever microscale simulator is provided. Roberts and Kevrekidis (2005, 2007) developed patch coupling conditions to ensure high order accuracy in the gap-tooth scheme for a class of dissipative systems. One aim here is to show that analogous coupling conditions also work for weakly or non-dissipative wave dynamics.

Section 2 establishes a microscale simulation of wave dynamics on a staggered grid. The microscale simulation is a pared down version of the equations of the shallow water dynamics because we plan to later adapt the approach to shallow water problems such as turbulent floods and tsunamis.

As a basic example of patch simulation of wave-like dynamics with a staggered grid, consider the simple linear wave PDEs

$$
\frac{\partial h}{\partial t}=-\frac{\partial u}{\partial x} \text { and } \frac{\partial u}{\partial t}=-\frac{\partial h}{\partial x}
$$

where in a water wave application $h$ and $u$ would represent the fields of 'water depth' and 'fluid velocity'. We aim to simulate the evolution of the fields $h(x, t)$ and $u(x, t)$, periodic in the $x$ direction, on the macroscale length $L=2 \pi$. Figure 1 exhibits a patch simulation of equation (1) through microscale simulation on $m=10$ patches, each of width $l=\pi / 15$, with macroscale spacing $D=\pi / 5$. No computations were performed on the empty space between the patches. Each patch appearing in Figure 1 integrates a spatial discretisation of the PDEs (1) on a microscale spatial grid of $n=9$ points (not counting the boundary points within each patch), with microscale grid spacing $d=D / 30=\pi / 150$. The microscale spatial discretisation of equation (1) represents a finely detailed model or particle simulation which is assumed far too expensive to use over a large domain (Kevrekidis et al., 2003, e.g.). Section 3 introduces one scheme to couple such patches of microscale simulators by interpolation in order to simulate the macroscale wave-like dynamics to varying degrees of accuracy.

Figure 1 and a supplementary movie ${ }^{1}$ show that microscale waves oscillate

${ }^{1}$ http://anziamj . austms . org.au/ojs/index.php/ANZIAMJ/article/ downloadSuppFile/6137/1111 

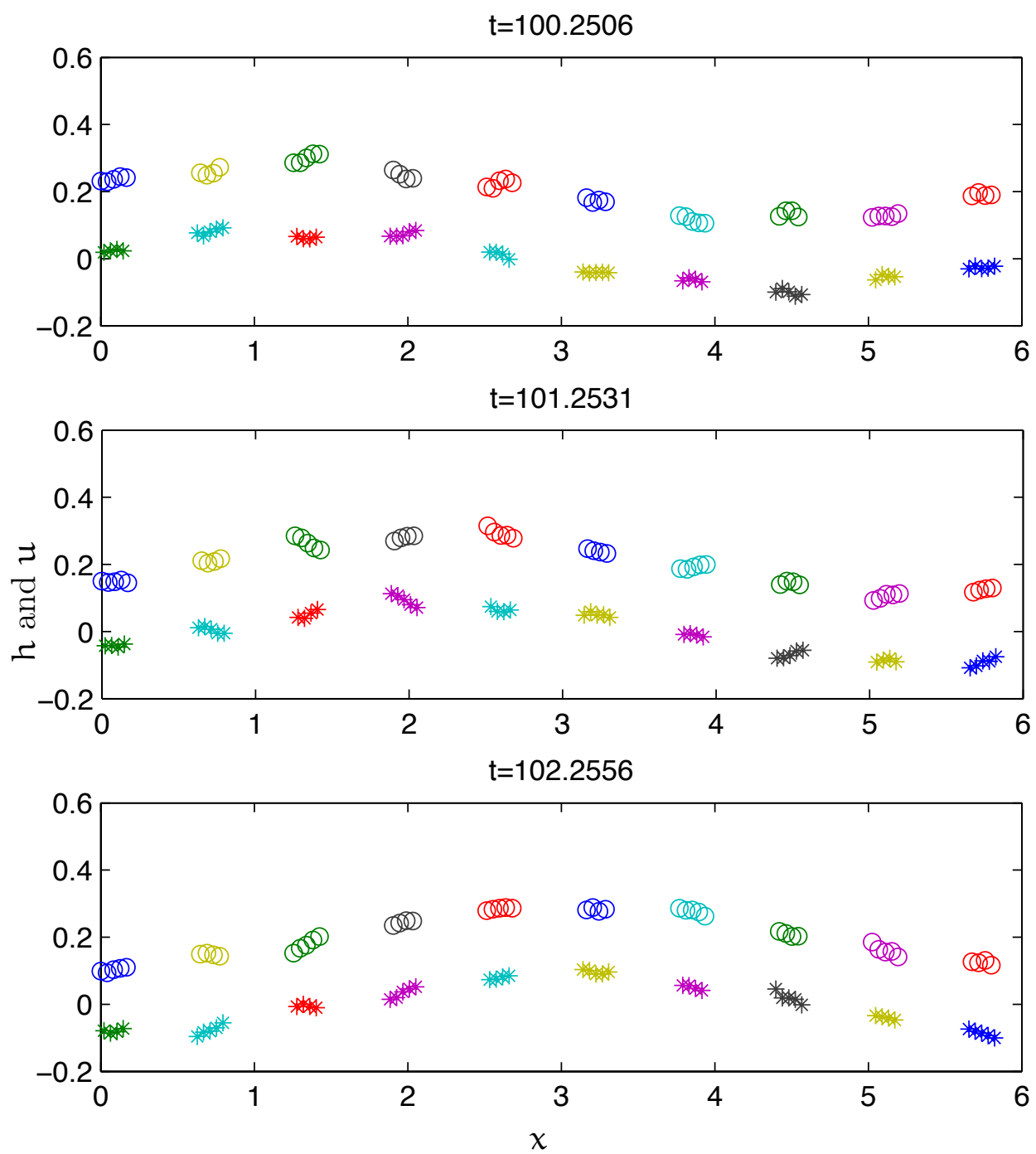

Figure 1: Patch simulation of the depth $h$ (circles) and velocity $u$ (stars) in equation (1) on $[0,2 \pi]$ at fixed time $t=100.25,101.25$ and 102.25. There are $m=10$ patches and $n=9$ microscale grid points on each patch (excluding patch boundaries). The patches are coupled by simple linear interpolation. A supplementary movie shows more details of the patch simulation of equation (1). 
rapidly in comparison to the macroscale waves. Nonetheless the macroscale waves appear to propagate unscathed by the bath of microscale vibrations. Section 4 explores the eigenvalues of the implemented gap-tooth scheme to support the veracity of the simulations in Figure 1 and the movie.

\section{The microscale simulator}

This section describes the microscale simulation of the wave-like dynamics in each patch. Staggered microscale grids are used to represent the fields of depth $\mathrm{h}$ and velocity $\boldsymbol{u}$.

This section considers the wave-like dynamics of two fields analogous to the water depth $h(x, t)$ and water velocity $\mathfrak{u}(x, t)$. The one dimensional linear governing equations analogous to the mass and momentum equations are

$$
\begin{aligned}
& \frac{\partial h}{\partial t}=-\frac{\partial u}{\partial x}, \\
& \frac{\partial u}{\partial t}=-\frac{\partial h}{\partial x}-v_{0} u+v_{2} \frac{\partial^{2} u}{\partial x^{2}},
\end{aligned}
$$

where the parameters $v_{0}$ and $v_{2}$ are constant coefficients analogous to bed friction and fluid viscosity respectively.

The first task is to code a microscale simulator of the linear equations (2) and (3) via a spatial discretisation on the microscale. As shown schematically in Figure 2 we now focus on the simulation within the jth patch. Within each patch we use a staggered grid of $n$ interior grid points and two boundary points on each patch. The microscale grid spacing is $d=l /(n+1)$, where $l$ is the width of each patch. Figure 3 shows the staggered grid for the depth $h_{j, i}$ and velocity $\mathfrak{u}_{j, i}$ at the $i$ th point of the micro-grid of a patch: we use two different patches depending upon whether the patch index $j$ is odd or even. Thus the microscale simulator discretises equations (2) and (3) on the interior 


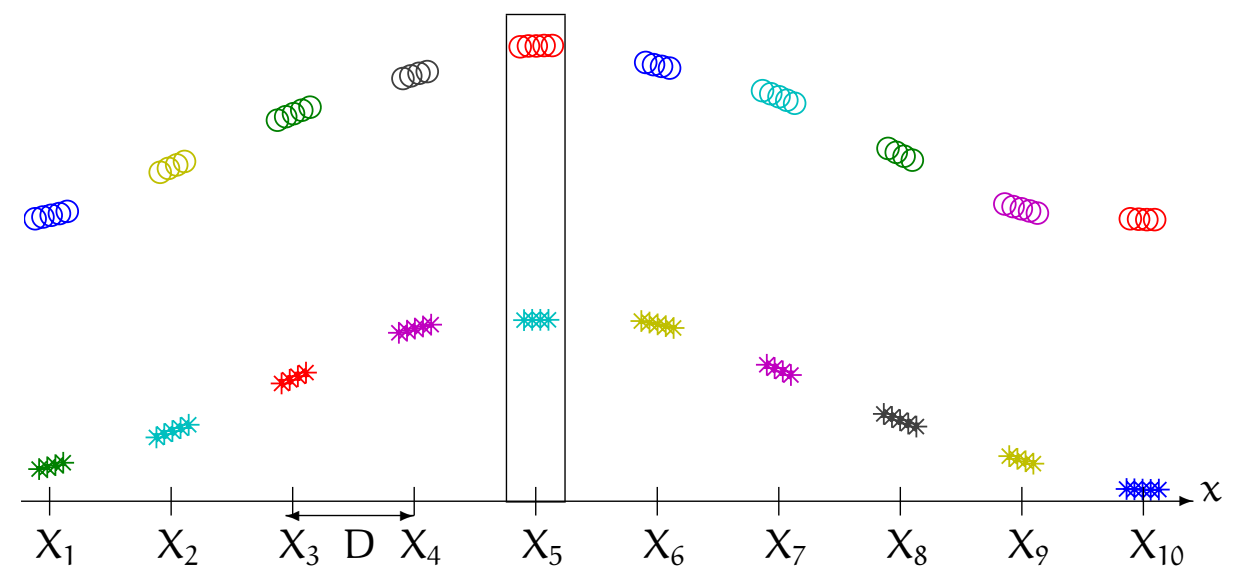

Figure 2: the rectangle indicates that we now zoom in on the jth microscale patch centred on a macroscale grid point $X_{j}$ for a macroscale grid of spacing $D$.

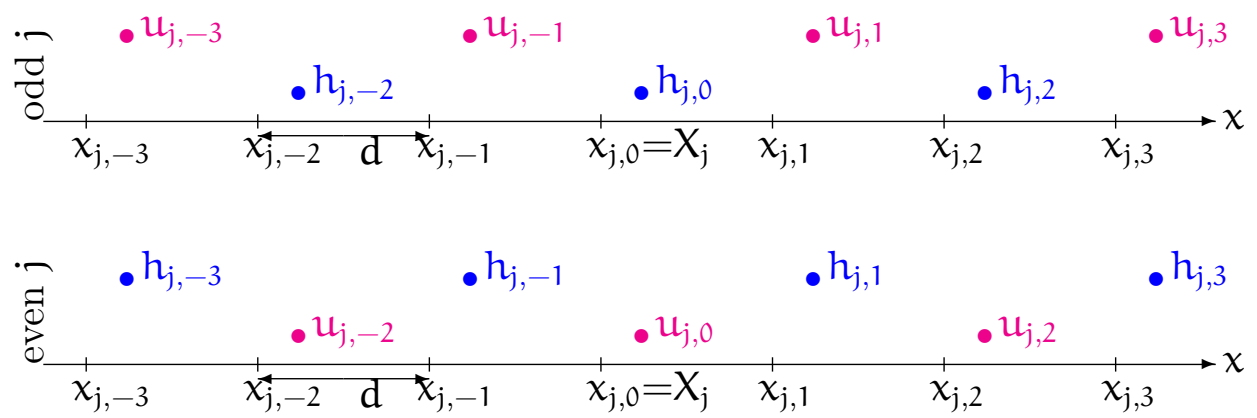

Figure 3: Scheme of the staggered grid points of the depth $h_{j, i}$ (blue points) and velocity $u_{j, i}$ (magenta points) at the ith micro-grid point on the odd $j$ th patch (top) and the even jth patch (bottom). This diagram show the cases for $n=5$ interior grid points in each patch. 


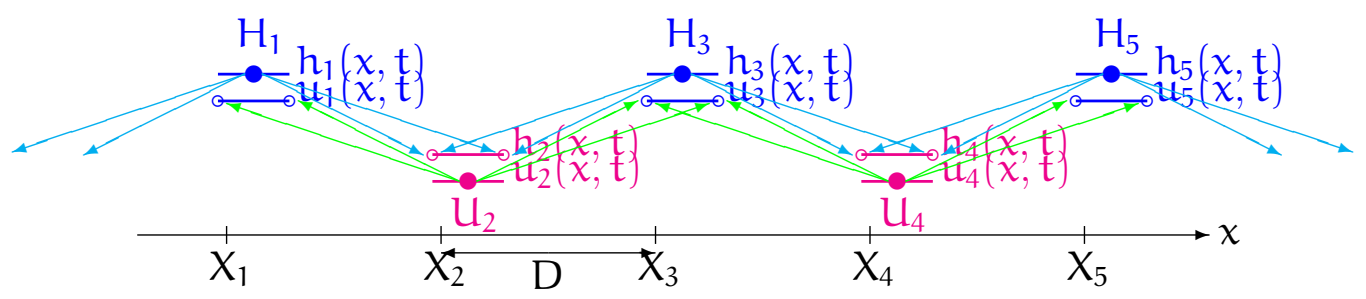

Figure 4: Scheme of the staggered macroscale grid of patches.

of the jth patch as

$$
\begin{aligned}
\frac{d h_{j, i}}{d t} & =-\frac{u_{j, i+1}-u_{j, i-1}}{2 d}, \\
\frac{d u_{j, i}}{d t} & =-\frac{h_{j, i+1}-h_{j, i-1}}{2 d}-v_{0} u_{j, i}+v_{2} \frac{u_{j, i+2}-2 u_{j, i}+u_{j, i-2}}{4 d^{2}},
\end{aligned}
$$

The movie sampled in Figure 1 was generated by such microscale simulations being coupled together as described in the next section.

Roberts and Kevrekidis (2005) described the gap-tooth scheme applied to the nonlinear Burgers' equation. Analogously, in future work we plan to implement nonlinear dynamics in the microscale model. Then we will proceed to simulate nonlinear water wave dynamics.

\section{Couple microscale patches across gaps}

This section aims to describe the wave-like dynamics via a macroscale staggered grid of patches. We couple the patches by interpolating information from neighbouring patches into boundary values for each of the microscale patch simulators of Section 2.

As shown schematically in Figure 4, let each of $m$ patches be centred on equispaced macroscale grid points $x=X_{j}=j D$, where $D=L / m$ is the macroscale 
spacing and $L$ is the length of the whole domain. Each patch has relatively small width $l$. Let each patch around a macroscale grid point $X_{j}$ execute a microscale simulation of the wave-like dynamics. Section 2 introduced that two different microscale simulations are defined for odd and even $\mathfrak{j}$, and Figure 4 illustrates these simulations alternating to form a macroscale staggered grid. The edge of each patch is a distance $l / 2$ from its macroscale grid point. Define the scale ratio $r=l /(2 \mathrm{D})$ to characterise the size of each patch relative to the distance between neighbouring patches: when $r=1 / 2$ the neighbouring patches meet as in holistic discretisation by Roberts (1999); when $r=1$ the patches overlap which establishes a slow manifold view of nonlinear wave-like dynamics. When the ratio $r$ is small, the patches form a relatively small part of the physical domain to provide a computationally efficient scheme for multiscale simulation.

As also shown in Figure 4, let macroscale grid values $H_{j}=h_{j}\left(X_{j}, t\right)$ for even $j$ and $u_{j}=u_{j}\left(X_{j}, t\right)$ for odd $j$. We interpolate these macroscale grid values to provide boundary values for each microscale patch. Computationally, all microscale simulators could execute in parallel with the only necessary communication between patches being these macroscale grid values. ${ }^{2}$ To interpolate the macroscale grid values we use finite difference operators. Define a shift operator $\varepsilon$ over two patches, for example, $\varepsilon h(x, t)=h(x+2 D, t)$ and $\varepsilon U_{j}=U_{j+2}$. Note the identities for discrete operators (Roberts and

\footnotetext{
${ }^{2}$ Such computational communication is synchronous as analysed herein. However, asynchronous computation and communication could be modelled by including a couple of intermediary variables into the dynamics between the actual macroscale value and its use as a patch boundary value. For example, for each macroscale variable $\mathrm{H}_{j}$ one could define two intermediaries, $H_{j}^{\prime}$ and $H_{j}^{\prime \prime}$ say, with dynamics $\tau d H_{j}^{\prime} / d t=-H_{j}^{\prime}+H_{j}$ and $\tau d H_{j}^{\prime \prime} / d t=-H_{j}^{\prime \prime}+H_{j}^{\prime}$ for some mean delay $2 \tau$ caused by asynchronous computation. Then the model to analyse is one where variables $\mathrm{H}_{j}^{\prime \prime}$, instead of $\mathrm{H}_{j}$, were interpolated to provide patch boundary values.
} 
Kevrekidis, 2005, 2007, e.g.),

$$
\begin{aligned}
\text { centred mean } & \mu=\frac{1}{2}\left(\varepsilon^{1 / 2}+\varepsilon^{-1 / 2}\right), \\
\text { centred difference } & \delta=\varepsilon^{1 / 2}-\varepsilon^{-1 / 2}, \\
\text { shift } & \mathcal{E}=1+\mu \delta+\frac{1}{2} \delta^{2} .
\end{aligned}
$$

Now, the edges of the $j$ th patch are at $x=X_{j} \pm(r / 2) 2 D$ which is a fraction $r / 2$ of a shift from the macroscale grid point $X_{j}$. The corresponding shift

$$
\varepsilon^{ \pm r / 2}=\left(1 \pm \mu \delta+\frac{1}{2} \delta^{2}\right)^{r / 2}=\frac{\mu}{\sqrt{1+\delta^{2} / 4}}\left(1 \pm \mu \delta+\frac{1}{2} \delta^{2}\right)^{r / 2}
$$

as $\mu^{2}=1+\frac{1}{4} \delta^{2}$. Expanding (9) in the Taylor series in small difference $\delta$, and replacing $\mu^{2}$ by $1+\frac{1}{4} \delta^{2}$, the shifts to the edges of a patch are

$$
\begin{aligned}
\mathcal{E}^{ \pm r / 2}= & {\left[\mu \pm \frac{1}{2} r \delta+\frac{1}{8}\left(-1+r^{2}\right) \mu \delta^{2} \pm \frac{1}{48}\left(-r+r^{3}\right) \delta^{3}\right.} \\
& +\frac{1}{384}\left(9-10 r^{2}+r^{4}\right) \mu \delta^{4} \pm \frac{1}{3840}\left(9 r-10 r^{3}+r^{5}\right) \delta^{5} \\
& +\frac{1}{46080}\left(-225+259 r^{2}-35 r^{4}+r^{6}\right) \mu \delta^{6} \\
& \left. \pm \frac{1}{645120}\left(-225 r+259 r^{3}-35 r^{5}+r^{7}\right) \delta^{7}\right]+\mathcal{O}\left(\delta^{8}\right) .
\end{aligned}
$$

This expansion then empowers us to express values on the edges of the jth patch, at $x=X_{j} \pm r D$, in terms of the macroscale grid values $H_{j}$ and $U_{j}$. Thus for odd/even $\mathfrak{j}$ we set the microscale, patch boundary, 'depth'/'velocity' as

$$
\begin{aligned}
\left.\left(h_{j}, u_{j}\right)\right|_{x=x_{j} \pm r D} \approx & {\left[\gamma \mu \pm \frac{1}{2} \gamma r \delta+\frac{1}{8} \gamma^{2}\left(-1+r^{2}\right) \mu \delta^{2} \pm \frac{1}{48} \gamma^{2}\left(-r+r^{3}\right) \delta^{3}\right.} \\
& +\frac{1}{384} \gamma^{3}\left(9-10 r^{2}+r^{4}\right) \mu \delta^{4} \pm \frac{1}{3840} \gamma^{3}\left(9 r-10 r^{3}+r^{5}\right) \delta^{5} \\
& +\frac{1}{46080} \gamma^{4}\left(-225+259 r^{2}-35 r^{4}+r^{6}\right) \mu \delta^{6} \\
& \left. \pm \frac{1}{645120} \gamma^{4}\left(-225 r+259 r^{3}-35 r^{5}+r^{7}\right) \delta^{7}\right]\left(H_{j}, u_{j}\right),
\end{aligned}
$$

where the parameter $\gamma$ conveniently labels the spatial extent of the interpolation. The interpolation (11) couples the patches together. 
One obtains various accuracies by truncating the coupling (11) to various orders in the label $\gamma$. For example, truncating to errors $\mathcal{O}\left(\gamma^{2}\right)$ gives linear interpolation, $h_{j}=\left(\mu \pm \frac{1}{2} r \delta\right) H_{j}$, from the nearest neighbour patches as Figure 4 illustrates. Whereas truncating to errors $\mathcal{O}\left(\gamma^{3}\right)$ gives cubic interpolation from nearest and next nearest patches. The details recorded in the coupling (11), that is to errors $\mathcal{O}\left(\gamma^{5}\right)$, are equivalent to interpolating a seventh order polynomial through the eight neighbouring macroscale values $\mathrm{H}_{j \pm 1}, \mathrm{H}_{j \pm 3}, \mathrm{H}_{j \pm 5}$ and $\mathrm{H}_{j \pm 7}$. We expect that such high order interpolation achieves high order accuracy as it does for dissipative systems (Roberts and Kevrekidis, 2005, e.g.).

Numerical simulations of the wave-like system (2)-(3) with coupling condition (11) verifies that the proposed coupling works. Figure 5 displays a gap-tooth simulation of the depth $h$ and velocity $\mathfrak{u}$ governed by a microscale discretisation of (2)-(3) with $m=10$ patches and $n=9$ microscale grid points on each patch. A fifth order polynomial is used from the coupling (11), that is to errors $\mathcal{O}\left(\gamma^{4}\right)$ in the label $\gamma$. The initial condition (blue), with some random noise within each patch, smooths rapidly by 'diffusion' $v_{2} u_{x x}$ to a quasi-equilibrium (green). A supplementary movie ${ }^{3}$ shows that the macroscale wave decays slowly due to the 'bed friction' $v_{0} u$ to a near steady state at time $t \approx 200$.

The multiscale modelling reduces the numerical cost of the simulations for large enough scale separation between the microscale computation and the resolved macroscale structures. For example, when the microscale grid spacing is $\mathrm{d}=0.0026$, our Matlab multiscale code with ten patches on the domain takes a computation time of 0.38 seconds in one period, whereas a corresponding Matlab code that resolves the microscale dynamics over the whole domain takes 21 seconds. In this example, the complete microscale simulation is 56 times slower to compute than our multiscale patch simulation. In more spatial dimensions we expect the computational time savings to be much larger as the fraction of space occupied by patches is expected to be much

${ }^{3}$ http://anziamj . austms . org.au/ojs/index.php/ANZIAMJ/article/ downloadSuppFile/6137/1112 


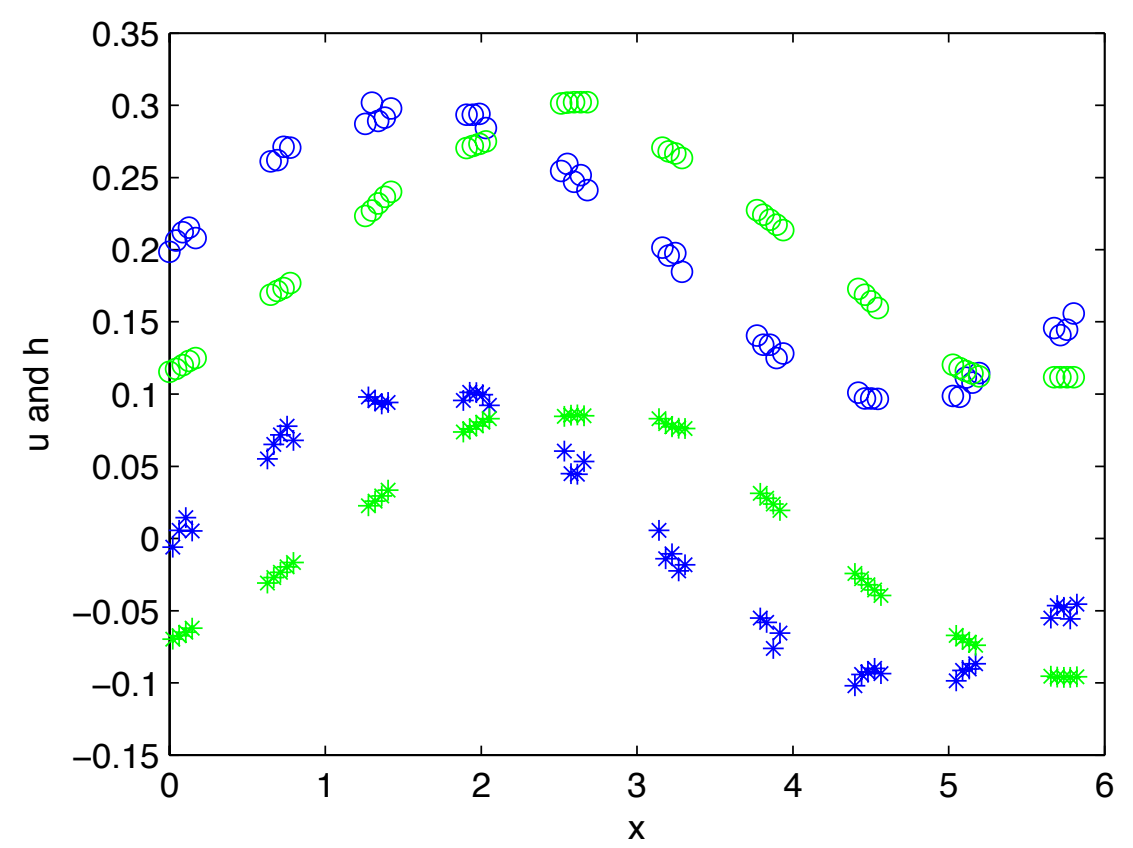

Figure 5: Patches simulations of the depth $\boldsymbol{h}$ (circles) and velocity $\mathfrak{u}$ (stars) in equations (2) and (3) on $[0,2 \pi]$ via $m=10$ patches and $n=9$ microscale grid points on each patch at time $t=0$ (blue) and $t=1$ (green). The scale ratio $r=1 / 6$, and the coefficients $v_{0}=0.01$ and $v_{2}=0.03$. A supplementary movie shows the simulations over the time $t=0: 200$.

smaller in higher dimensions.

\section{Linear analysis of the coupled dynamics}

This section linearly analyses the proposed gap-tooth multiscale modelling. Our focus is on the performance of the coupling conditions so we analyse the wave-like PDEs (2)-(3), and the numerical eigenvalues of its microscale 
discretisation (4)-(5), and both on patches coupled by (11).

On the $j$ th patch assume the fields of the depth $h_{j}$ and velocity $u_{j}$ have solutions in the forms of

$$
h_{j}(x, t)=C_{h} e^{\lambda t+i(k j+\ell \xi)} \quad \text { and } \quad u_{j}(x, t)=C_{u} e^{\lambda t+i(k j+\ell \xi)},
$$

for microscale space variable $\xi=\left(x-X_{j}\right) / D$, the complex growth rate $\lambda$, macroscale wavenumber $k$, and microscale wavenumber $\ell$. These wavenumbers are both defined relative to the macroscale grid spacing D. Substitute the solution form (12) into the equations (2) and (3), factor $e^{\lambda t+i(k j+\ell \xi)}$ and obtain

$$
\lambda C_{h}=-i \frac{\ell}{D} C_{u} \quad \text { and } \quad \lambda C_{u}=-i \frac{\ell}{D} C_{h}-v_{0} C_{u}-v_{2} \frac{\ell^{2}}{D^{2}} C_{u} .
$$

For non-trivial solutions for $C_{h}$ and $C_{\mathfrak{u}}$ the complex growth-rate must satisfy

$$
\lambda^{2}+\left(v_{0}+v_{2} \frac{\ell^{2}}{\mathrm{D}^{2}}\right) \lambda+\frac{\ell^{2}}{\mathrm{D}^{2}}=0
$$

which determines the dispersion relationship between the growth rate $\lambda$ and microscale wavenumber $\ell$.

The microscale wavenumber $\ell$ is then determined by the coupling conditions as a function of macroscale wavenumber $k$. A supplementary file ${ }^{4}$ lists a computer algebra ${ }^{5}$ program to use the coupling conditions (11) to derive the high order characteristic equation

$$
\pm \sin \ell r+r \sin k+\frac{1}{6}\left(r-r^{3}\right) \sin ^{3} k+\frac{1}{120}\left(9 r-10 r^{3}+r^{5}\right) \sin ^{5} k=\mathcal{O}\left(k^{7}\right) .
$$

Expanding a small microscale wavenumber $\ell$ in equation (15) in a Taylor series in the macroscale wavenumber $k$, we obtain from the explicitly given terms that

$$
\ell= \pm k+\frac{1}{5040}\left(-225+259 r^{2}-35 r^{4}+r^{6}\right) k^{7}+\mathcal{O}\left(k^{9}\right) .
$$

\footnotetext{
${ }^{4}$ http://anziamj .austms .org.au/ojs/index .php/ANZIAMJ/article/ downloadSuppFile/6137/1110

${ }^{5}$ http://www.reduce-algebra.com
} 
That is, to the order of analysis used to obtain (15) the coupling conditions (11) ensure that the microscale wavenumber agrees precisely with the macroscale wavenumber. This agreement ensures the high order accuracy of the gap-tooth scheme with these coupling conditions.

The characteristic equation (15) also has solutions with non-small $\ell$. For macroscale wavenumber $k=0$, these are $\ell=n \pi / r$ for integer $n$. For such large microscale wavenumber $\ell$, from (14) the growth-rate $\lambda$ has large imaginary part indicating rapid microscale oscillations/waves within patches. These rapid microscale waves are not of interest to the macroscale dynamicsoften microscale dissipation will damp them as seen in our movies. Importantly though, and even without dissipation, for non-zero macroscale wavenumber $k$ one can see that the solutions $\ell$ of the characteristic equation (15) will remain real. Consequently, the coupling conditions (11) maintain the microscale waves as waves; the coupling conditions do not turn the microscale waves into unstable modes that would wreck the macroscale simulation.

Numerical eigenvalues of the dynamics confirms the stability of the gap-tooth scheme with coupling conditions (11). Consider the microscale simulator (4) and (5) with the coupling conditions (11) in a fifth polynominal. The fields $h_{j, i}=1$ and $u_{j, i}=0$, say, is a steady state. We characterise the dynamics in the neighbourhood of this the steady state via the spectrum of the Jacobian. Numerical differentiation of the simulation function gives a sufficiently good approximation to the Jacobian, then standard routines compute the complete spectrum of eigenvalues. Figure 6 plots the real (growth rate) and imaginary part (frequency) of the numerical eigenvalues about this steady state for one set of parameters. The arcsinh scaling of the two axes provides a quasilogarithmic non-uniform scaling of the data. Figure 6 shows several eigenvalues with small $\mathfrak{R} \lambda$ - these are the macroscale waves which decay slowly through 'bottom friction' as they propagate.

The figure also shows many eigenvalues with large imaginary and real parts: these represent microscale waves within the patches that decay rapidly through microscale dissipation. Such rapid microscale waves, and their decay, is seen 


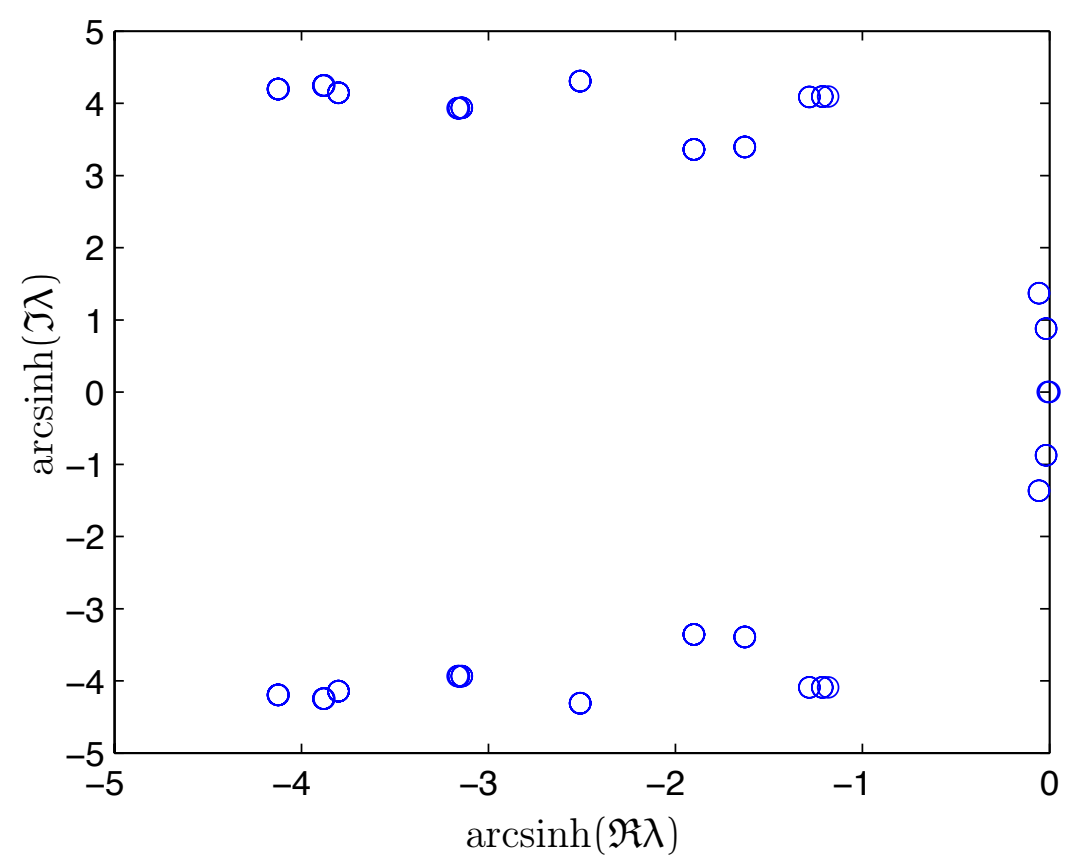

Figure 6: Distribution of the real and imaginary parts of the numerical eigenvalues for equations (4) and (5) with $\mathrm{m}=10$ patches and $\mathrm{n}=9$ microscale grid points on each patch. The length scale ratio $r=1 / 6$ and the coefficients $v_{0}=0.01$ and $v_{2}=0.03$. There are 44 complex conjugate pairs of values, and another two real values 0 and -0.1 .

in the initial transients shown in a supplementary movie. ${ }^{6}$

The gap in the growth rate $\mathfrak{R} \lambda$, shown in Figure 6 as the gap in $\operatorname{arcsinh} \mathfrak{R} \lambda$ between 0 and -1 , is interesting. This gap indicates that the set of macroscopic waves form a slow subspace, $\mathfrak{R} \lambda \approx 0$, among the multiscale dynamics of the waves on the coupled patches. We plan further research into nonlinear wave-like systems to use this slow subspace to establish the existence of a slow

\footnotetext{
${ }^{6}$ http://anziamj.austms.org.au/ojs/index.php/ANZIAMJ/article/ downloadSuppFile/6137/1112
} 
Table 1: Numerical eigenvalues for the macroscale wave modes from Figure 6 for $m=10$ and $m=14$ patches; compare with exact eigenvalues from equation (14) for the linear dynamics about the steady state.

\begin{tabular}{ccccc}
$\ell / D$ & multiplicity & $\lambda$, eqn $(14)$ & $\lambda, m=10$ & $\lambda, m=14$ \\
\hline 0 & one & $-0.000,-0.010$ & $0,-0.010$ & $0,-0.010$ \\
\pm 1 & two & $-0.020 \pm 0.998 i$ & $-0.020 \pm 0.998 i$ & $-0.020 \pm 0.999 i$ \\
\pm 2 & two & $-0.065 \pm 1.999 i$ & $-0.056 \pm 1.836 i$ & $-0.063 \pm 1.968 i$
\end{tabular}

manifold of the nonlinear macroscale wave dynamics (Carr, 1981; Roberts, 1988, e.g.).

Table 1 lists the macroscale numerical eigenvalues in Figure 6 and the exact eigenvalues from equation (14) about the steady sate. The numerical eigenvalues are close to the exact eigenvalues. Table 1 shows the conservation of fluid (eigenvalue zero) and the decay of bulk velocity due to bed friction (eigenvalue -0.010 ) are correct to at least three decimal places. The longest wave mode on the domain, $\ell / D= \pm 1$, which in the absence of friction would have frequency one, also has its eigenvalue correct to three decimal places. Numerical tests show that when the number of patches increases, the numerical eigenvalues become closer to the analytical eigenvalues, as shown in Table 1. For example, for the wavenumber $\ell / D= \pm 2$ mode, upon increasing the number of patches from $m=10$ to $m=14$ we find the eigenvalues $\lambda$ change from $-0.056 \pm 1.836 i$ to $-0.063 \pm 1.968 i$, which are significantly more accurate when compared to the exact eigenvalues $-0.065 \pm 1.999 i$.

\section{Conclusion}

We invoked a staggered grid to discretely model both the microscale and macroscale simulators of wave-like dynamics. Classic polynomial interpolation underlies the coupling of patches proposed in Section 3 to form a gap-tooth scheme. The resultant numerical simulations indicated that this could be 
a useful scheme for wave-like dynamics: movies of such simulations are in the supplementary files. Numerical simulations currently underway indicate that the scheme also works for nonlinear wave-like dynamics. Section 4 reported algebraic analysis and numerical determination of eigenvalues that both confirm the accuracy of the proposed gap-tooth scheme for wave-like dynamics. In particular, Figure 6 shows the clear separation between the dynamics of the macroscale waves of interest, and the microscale waves within each patch. Future work will focus on the theoretical accuracy of nonlinear wave simulation, in one or several dimensions, and their application to shallow water flow. This kind of multiscale modelling should also be applicable to modelling large scale turbulence floods and tsunamis (Roberts et al., 2008, e.g.) by empowering us to use a microscale turbulence model in a macroscale simulation.

Acknowledgements Parts of this research was supported by the Australian Research Council grant DP120104260 in collaboration with Yannis Kevrekidis of Princeton University.

\section{References}

Carr, J. (1981), Applications of centre manifold theory, Vol. 35 of Applied Math. Sci, Springer-Verlag. C167

Hou, T. Y., Yang, D. and Ran, H. (2008), 'Multiscale analysis and computation for the three-dimensional incompressible Navier-Stokes equations', Multiscale Modelling and Simulation 6(4), 1317-1346. doi:10.1137/070682046. C154

Hu, X. (2012), Multiscale Modeling and Computation of 3D Incompressible Turbulent Flows, $\mathrm{PhD}$ thesis, California Institute of Technology.

http://resolver. caltech. edu/CaltechTHESIS : $05302012-081356007$.

C154 
Kevrekidis, I. G., Gear, G. W., Hyman, J. M., Kevrekidis, P. G., Runborg, O. and Theodoropoulos, C. (2003), 'Equation-free, coarse-grained multiscale computation: enabling microscopic simulators to perform system-level analysis', Comm. Math. Sci. 1(4), 715-762.

http://projecteuclid.org/euclid.cms/1119655353. C154, C155

Roberts, A. J. (1988), 'The application of centre-manifold theory to the evolution of systems which vary slowly in space', J. Austral. Math. Soc. Ser. B 29, 480-500. doi:10.1017/S0334270000005968. C167

Roberts, A. J. (1999), 'Holistic finite differences ensure fidelity to Burger's equation'. http://adsabs. harvard.edu/abs/1999chao.dyn. .1011R. C160

Roberts, A. J., Georgiev, D. J. and Strunin, D. V. (2008), Model turbulent floods with the Smagorinsky large eddy closure, Technical report. http://arxiv.org/abs/0805.3192. C168

Roberts, A. J. and Kevrekidis, I. G. (2005), 'Higher order accuracy in the gap-tooth scheme for large-scale dynamics using microscopic simulators', ANZIAM Journal 46, C637-C657.

http://www.austms.org.au/Publ/Jamsb/. C154, C155, C159, C160, C162

Roberts, A. J. and Kevrekidis, I. G. (2007), 'General tooth boundary conditions for equation free modeling', SIAM Journal on Scientific Computing 29(4), 1495-1510. doi:10.1137/060654554. C155, C161

Samaey, G., Roberts, A. J. and Kevrekidis, I. G. (2009), Equation-free computation: an overview of patch dynamics, Vol. 8 of Multiscale Methods, Oxford Scholarship Online Monographs. doi:10.1093/acprof:oso/9780199233854.003.0008. C154

Samaey, G., Roose, D. and Kevrekidis, I. G. (2005), 'The gap-tooth scheme for homogenization problems', Multiscale Modelling and Simulation

4, 278-306. doi:10.1137/030602046. C154 


\section{Author addresses}

1. Meng Cao, School of Mathematical Sciences, University of Adelaide, South Australia 5005, Australia.

mailto:meng. cao@adelaide.edu.au

2. A. J. Roberts, School of Mathematical Sciences, University of Adelaide, South Australia 5005, Australia.

mailto:anthony.roberts@adelaide.edu.au 